SLAC-PUB-8476

June 2000

\title{
On The Shape of the Betatron Side-Band
}

\author{
S. Heifets, H.U. Wienands
}

Presented at the 7th European Particle Accelerator Conferences (EPAC 2000),

Vienna, Austria, 6/26/2000-6/30/2000

Stanford Linear Accelerator Center, Stanford University, Stanford, CA 94309

Work supported by Department of Energy contract DE-AC03-76SF00515. 


\section{Abstract}

The shape of the betatron side-band is sensitive to the transverse rms size of colliding bunches. It is shown in this note that the transverse rms of the beam can be inferred from the shape of the betatron line. Analysis is presented which takes into account the tilt angle of the beams at the collision point and their offset. We discuss other effects which can change the shape of the betatron line in the spectrum.

\section{Introduction}

The beam spectrum is one of the main tool of the beam diagnostics. We would like to emphasize that spectra can not only provide frequencies of the beam motion, but the shape of the lines can be used also to define beam parameters such as the rms transverse beam size of colliding beams. Measurements of the luminosity as function of the beam offset provides the

"capsigma" rms $\Sigma=\sqrt{\sigma_{1}^{2}+\sigma_{2}^{2}}$ of individual beams. The synchrotron light beam monitor may require calibration which is not straightforward. Unless dedicated diagnostics is used (as at KEK), the ratio of $\sigma_{1} / \sigma_{2}$ is undefined and it wouldbe useful to have an independent way to measure $\sigma_{1,2}$.

Power spectra were taken at the PEP-II B-factory in collision of the 9.1 $\mathrm{GeV}$ electron beam (HER) with $3 \mathrm{GeV}$ positron beam (LER) using the HP8940A spectrum analyzer. The betatron oscillations at low beam currents were excited by the random noise source of the spectrum analyzer. Examples of the spectra are given in Fig. ??. The noise of the background was numerically subtracted to obtain spectra shown in the figures.

The numbers in the figures are (HER $\mathrm{x}$ LER) beam currents in $\mathrm{mA}$. The colliding beams in the measurements had 12 equidistant bunches (11 colliding, one out of collisions). The spectra are asymmetric, quite sensitive to the beam currents, and different for two beams and for the horizontal/vertical motion.

The number of bunches in experiment was much smaller than nominal 1658 bunches to avoid complications of the coupled-bunch spectra and to minimize possible effect of trapped ions and photo-electrons cloud level of excitation was chosen as a trade-off between distortion of the spectra by the nonlinearity of motion with large amplitudes on one hand and the signal/noise ratio on the other. The beam spectrum taken with excitation level different by $10 \mathrm{db}$ have the same main features, Fig. ??, confirming that the excitation was low enough to avoid non-linear beam dynamics effects. 


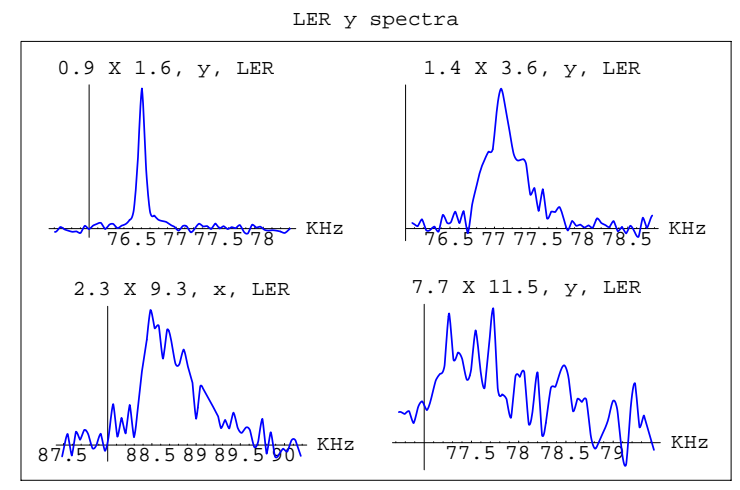

Figure 1: Shape of the betatron line in the experimental beam spectra in the PEP-II LER and HER.

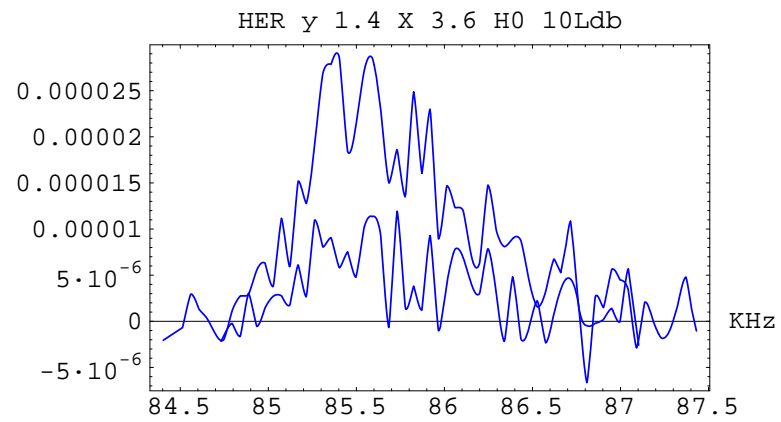

Figure 2: Beam spectra taken with two excitation level different by $10 \mathrm{db}$

\section{Signal}

The signal on the pick-up at the excitation frequency $\Omega$, is proportional to the effective impedance $R_{s}$ of the pick-up and the beam dipole moment given by the first harmonics $f(J)$ where $J$ is action variable, $<y(\Omega)>=$ $(1 / 2 i) \int d J \sqrt{2 J}\left[f^{*}(J,-\Omega)-f(J, \Omega)\right]$.

If 1$)$ there are no betatron resonances, 2) coupling of the excitation to the opposite bunch is weak, 3) additional to SR diffusion (quadratic in $\mathrm{f}$ ) is negligible, then the Fokker-Plank equation gives $f(J, \Omega)=-i F \sqrt{2 J}\left(d f_{0} / d J\right) /[\Omega+$ $Q(J)-i \Gamma]$, where $F$ is amplitude of excitation force, and $\Gamma$ is damping. The signal then is

$$
V_{s}(\Omega)=R_{s} F \int d J J\left(d f_{0} / d J\right) \Gamma /\left[(\Omega-Q(J))^{2}+\Gamma^{2}\right]
$$

. Here $f_{0}\left(J_{x}, J_{y}\right)$ is distribution function of the first beam normalized to one. The tune spread $Q(J)$ can be calculated taking into account the tilt angles and beam offsets. For realistic parameters, these dependencies are weak. There is correction to the exponent in $f_{0}$ due to beam-beam potential. It is of the order of the beam-beam parameters, and correction is small. 
Fig. 3 depicts one of results of calculations of the LER beam spectra in the vertical plane for 12 bunches in $1.4 \times 3.6 \mathrm{~mA}$ beams. The rms beam sizes at IP are shown in the figure. Other parameters corresponds to the experimental set up in Fig. $1, \beta_{x}=50 \mathrm{~cm}, \beta_{y}=1.5 \mathrm{~cm}$ at IP. Calculations reproduce asymmetry of the measured spectra and show that preferable HER beam size $\sigma_{y} \approx 4 \mu m$ is smaller than $\Sigma_{y} \sqrt{2}$ for equal beams.

$y-L E R, 2.3 \times 9.3$

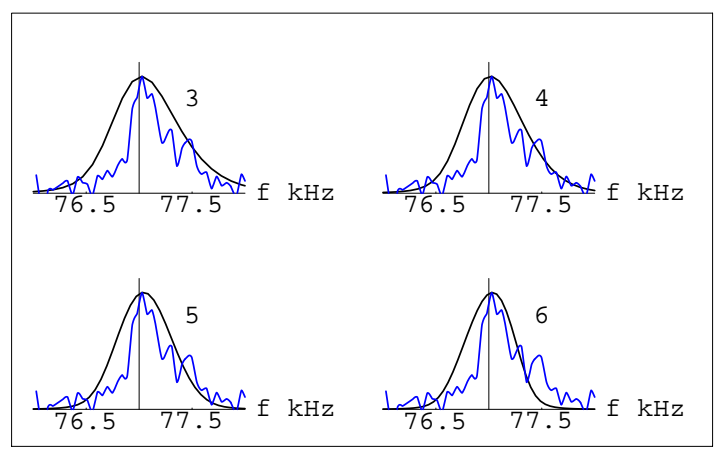

Figure 3: Calculated LER spectra in y -plane for $\sigma_{y, H E R}=3,4,5$, and $6 \mu \mathrm{m}$ at IP. $\Sigma_{x, y}$ are known from luminosity measurements, $\Sigma_{x}=228 \mu \mathrm{m}, \Sigma_{y}=$ $7.2 \mu \mathrm{m} . \sigma_{x}$ are taken equal for both beams. The beam currents $2.3 \times 9.3 \mathrm{~mA}$.

However, calculations for the HER failed to reproduce experimental spectra. For example, the line in Fig. 4 is much narrower than in the measurements and does not reproduce the wide shoulder at high frequencies. The discrepancy is even more noticeable for 12 bunches. The y-HER spectrum at $1.4 \times 3.6 \mathrm{~mA}$ shows two horn distribution which calculations fail to reproduce. At higher currents experimental spectra become even wider and noisier. It should be noted, that the amplitude of the HER signal is smaller than that for the LER spectra by a factor 8-9 (reflecting the difference in the beam energies), and is close to the noise level. Nevertheless, we tried to look for other possible mechanisms affecting beam spectra. This is discussed in the next section.

\section{Other mechanisms affecting beam spectrum.}

There are many mechanisms which may affect the shape of the betatron side-band in the beam spectrum.

- The amplitude dependence of the tune due to lattice nonlinearities is negligible small, the largest coefficient $d Q_{y} / d J_{x}=870 \mathrm{~m}$.

- Synchro-betatron coupling produces synchrotron side-bands at the distance $Q_{s}$ much larger than $\xi_{B B}$, and do not change the shape of the betatron line except the amplitude of the signal. 
$1.4 \times 3.6$

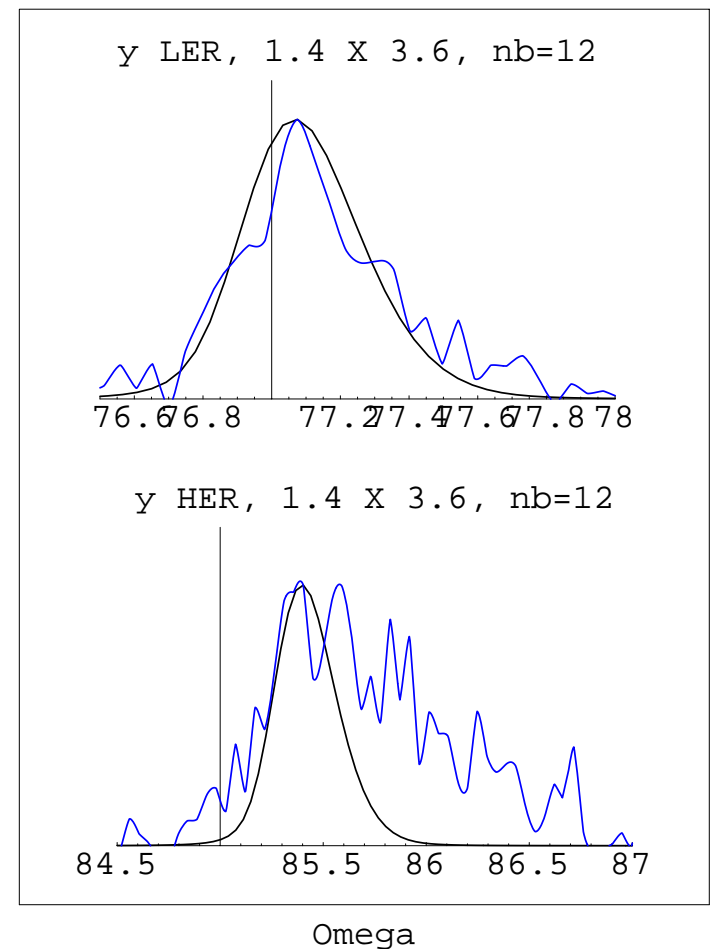

Figure 4: y-spectra of both beams. $\sigma_{x, L E R}=\sigma_{x, H E R}, \sigma_{y, H E R}=4 \mu m$, $\sigma_{y, L E R}=6 \mu \mathrm{m}$. No fitting is used in the position of the maximum. HER spectrum is much wider than expected from beam-beam tune spread.

- In the same way, the (absolute) chromaticity $\xi$ modifies the amplitude of the betatron side-band, but does not change the shape of the line.

- The width of the line is given by the SR and head-tail damping. For $\xi \simeq 2$,the head-tail damping time is longer than SR damping time and the total width of the betatron line is small compared with $\xi_{B B}$.

- The threshold of the microwave instability in PEP-II is by two order of magnitude higher than the transverse impedance of the rings.

- The $x-y$ global coupling may change the vertical beam size and the beam-beam tune spread in y-plane. The PEP-II global coupling is much smaller than beam-beam parameter $\xi_{B} B$ and effect of the coupling is additionally reduced by the $\mathrm{x}-\mathrm{y}$ tunes separation $\Delta f \simeq 10 \mathrm{KHz}$.

- Coupled-bunch mode-to-mode tune variation in the PEP-II is negligible small in the experiment.

- The beam-to-beam coupling can be enchanced for $Q_{x, H E R} \simeq Q_{y, L E R}$. The spectrum has a split maximum with two picks separated by $\Delta \Omega=$ 
$\sqrt{\lambda^{2} \xi_{B B}^{2}+\left(\omega_{x}-\omega_{y}\right)^{2}}$. However, the coupling coefficient $\lambda$ is of the order of the $\mathrm{x}-\mathrm{y}$ coupling or is given by the nonlinearity of the beambeam force. In the last case $\lambda \propto \sigma_{y} / \sigma_{x}$.In both cases effect is small compared to $\xi_{B B}$.

- The betatron side-band can be affected by a betatron resonances which modifies the distribution function in the vicinity of the resonance,see Fig. ??. In the lab frame, such a distribution is time dependent and is a superposition of azimuthal harmonics $f_{n}(J)$.A large number of harmonics are excited with amplitudes $\left|f_{n}(J)\right|^{2}=\left(\frac{m}{\pi n}\right)^{2} \sin ^{2}(n / m) \sqrt{1-p^{2}}$, where $p=\left|\partial Q\left(J_{r}\right) / \partial J_{r}\right| J-J_{r} \mid / \Delta Q .0<p<1$, and $\Delta Q$ is the width of the resonance. The resonance harmonics $n=1$ of the test beam directly affects the dipole signal, while harmonics $n=0$ due to resonance in the opposite beam affect the betatron side-band line changing the beam-beam induced tune spread. Our calculations show that although there is noticeable correction to $\Delta Q(J)$, the shape of the signal is affected very little, see Fig. ??.
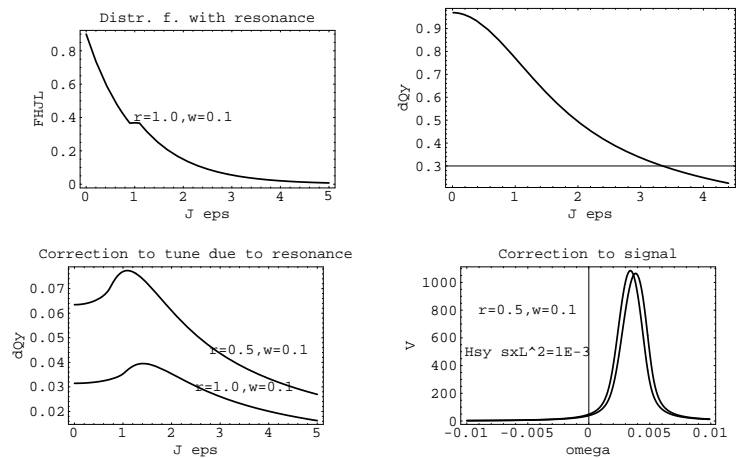

Figure 5: Effect of a resonance on the tune spread and shape of the side-band. 1D model

- Ions in the HER affect the spectrum. With few mA total HER current in 12 bunches, hydrogen ions are free, but CO ions are trapped. The beam current used in the experiment are below the threshold of fast ion instability. Ions produce tune shift proportional to the total number of ions in the ring $N_{i, t o t}$. For the equidistant bunches, the last is defined by the condition of neutrality or by the secondary ionization. The tune shift in the linear theory is large but the nonlinear terms tend to cancel it and the tune shift depends on the beam pipe aperture rather than transverse rms of the beam. The tune spread, generally, of the same order as the tune shift, depends on the details of the ion distribution function and is changed substantially by the space charge of accumulated ions. It is reasonable to expect in this limit broadening and large fluctuations of the beam spectra. However, de-trapping of 
the $\mathrm{CO}$ ions occurs at currents $I \simeq 8 \mathrm{~mA}$, while experimental spectra keep broadening with current.

- The density of electron cloud in the LER is limited by the space-charge effect. Additionally to the tune shift and spread, electron cloud may produce a fluctuating force. Random uncorrelated fluctuations of the number of electrons at each location induce rms

$$
<y^{2}>=(\pi / 2) c \tau_{d}\left(\frac{r_{0} R}{\gamma Q}\right)^{2} n_{e}
$$

where $n_{e}$ is the average density of the electron cloud. The LER transverse emittance growth affects tune spread and beam spectra in the HER. The vertical damping time, $\tau_{d}=1 / \tau_{S} R-1 / \tau_{e \gamma}$, and the rm$\mathrm{s}$ grow while growth rate $\tau_{e \gamma}$ of $\mathrm{e}-\gamma$ instability approaching the SR growth rate. Unfortunately, increase of the rms of the LER beam means smaller beam-beam tune spread in the HER and does not help with explaining the HER spectra.

- If the broad-band feedback has a reactive component then, by the full analogy with any impedance,it would produce additional tune spread. The noise of the feedback also can change the beam emittance and in this way change the tune spread. However, with the beam in collision, the transverse feedback was off.

\section{Conclusion}

Experimental spectra measured in the PEP-II B-factory for colliding beams display quite complicated shape of the betatron line. The line is asymmetric, wide, and both asymmetry and the width are current dependent. These effects can be explained by the dependence of the beam-beam tune spread on the transverse rms dimensions of the opposite beam. We present results of calculations which show that the proper choice of the transverse rms may explain the experimental spectra in the LER and the useful information on the beam size can be inferred from the shape of the line. However, spectra in the HER more noisy and wider than spectra in the LER and can not be explained in the same way.

Although it is true that the signal to noise ratio in the HER spectra is lower than that for the LER spectra, the effect is current dependent and, therefore, we don't think that it is pure instrumental or is a result of the tune jitter due to noise in power supply.

We present some analysis of other mechanisms which could affect spectra and would provide explanation for the experimental results. It seems, that only ions and, maybe, beam-beam resonances can explain the spectra. This will be verified in the future studies. 